\title{
Alternative Approach for Satellite Cloud Classification: Edge Gradient Application
}

\author{
Jules R. Dim ${ }^{1}$ and Tamio Takamura ${ }^{2}$ \\ ${ }^{1}$ Earth Observation Research Center/JAXA, 2-1-1 Sengen, Tsukuba, Ibaraki 305-8505, Japan \\ ${ }^{2}$ Center for Environmental Remote Sensing (CEReS), Chiba University, 1-33 Yayoi-cho, Inage-Ku, Chiba 263-8522, Japan
}

Correspondence should be addressed to Jules R. Dim; rosutando@yahoo.com

Received 17 February 2013; Revised 20 June 2013; Accepted 21 October 2013

Academic Editor: Ismail Gultepe

Copyright (C) 2013 J. R. Dim and T. Takamura. This is an open access article distributed under the Creative Commons Attribution License, which permits unrestricted use, distribution, and reproduction in any medium, provided the original work is properly cited.

Global atmospheric heat exchanges are highly dependent on the variation of cloud types and amounts. For a better understanding of these exchanges, an appropriate cloud type classification method is necessary. The present study proposes an alternative approach to the often used cloud optical and thermodynamic properties based classifications. This approach relies on the application of edge detection techniques on cloud top temperature (CTT) derived from global satellite maps. The gradient map obtained through these techniques is then used to distinguish various types of clouds. The edge detection techniques used are based on the idea that a pixel's neighborhood contains information about its intensity. The variation of this intensity (gradient) offers the possibility to decompose the image into different cloud morphological features. High gradient areas would correspond to cumulus-like clouds, while low gradient areas would be associated with stratus-like clouds. Following the application of these principles, the results of the cloud classification obtained are evaluated against a common cloud classification method based on cloud optical properties' variations. Relatively good matches between the two approaches are obtained. The best results are observed with high gradient clouds and the worst with low gradient clouds.

\section{Introduction}

The present study is motivated by the future launch of a new polar orbit satellite, the global change observation missionclimate (GCOM-C) carrying a visible and thermal infrared sensor, the second generation global imager (SGLI). The objectives of this satellite include the reduction of the Earth's radiation budget uncertainty. One of the major factors affecting this uncertainty is the change in cloud type amount [1]. To quantify such a change, a cloud type classification is needed. The existence of multiple satellite sensors' channels provides good opportunities for these cloud type classifications. In cloud remote sensing, the most frequently used channels for cloud classifications are in the visible and the infrared bands. For the visible bands, one of the most common classifications relies on the primary cloud property, that is, the cloud optical depth ([2]; Rossow et al., 2003) to distinguish cloud types. In the thermal infrared channels, the classifications often use thermodynamic properties of clouds as derived from splitwindow channels [3].

In the present study, a different approach from that often used in common classifications is proposed. This approach is based on the cloud top structure contrast. For its implementation, cloud top temperature (CTT) images derived from satellite thermal infrared observations are used. The CTT images are translated into visual features with enough contrast to allow for the distinction of different cloud types. To facilitate this distinction, edge detection techniques are applied on the CTT images. To capture and portray the variability of cloud shapes and appearance in satellite images, a cloud classification technique is expected to be less computationally demanding, show better differentiation of boundaries of varying clouds, use reasonably small agglomeration of cloud pixels, be robust to noise, maximize the signal-to-noise ratio, have good localization capacities, and so forth. Segmentation techniques among which are edge detection methods can be 
TABLE 1: Concept of cloud type classification using edge detection techniques.

\begin{tabular}{lccc}
\hline Cloud level & & Type of cloud & \\
\hline High cloud & Cirrus & Cirrostratus & Deep convection \\
Mid cloud & Altocumulus & Altostratus & Nimbostratus \\
Low cloud & Cumulus & Stratocumulus & Stratus \\
\hline Degree of structuring & Structured & & Nonstructured \\
& (High gradient occurrence) & (Low gradient occurrence) \\
\hline
\end{tabular}

appropriately used to satisfy these conditions. Edge methods used in the present study could play a prominent role in shape differentiations compared to region based or pixel based methods such as the K-means clustering.

Advanced steps in the implementation of segmentation techniques include some commonly used methods such as general clustering, simple thresholding, region-growing, distribution mask, or fixed histograms of gradients. For instance, in Dalal and Triggs $[4,5]$, locally normalized histogram of gradient orientations features is used to study feature sets for human detection. Sen and Pal [6] use a bilevel histogram thresholding methodology based on fuzzy and rough set theories to perform segmentation and edge extraction on grayscale and gradient magnitude images. Smith and Brady [7] describe edge and corner detection and structure preserving noise reduction for low level image processing. Shashua et al. [8] use fixed subregions to extract vector features in pedestrian detection. Suard et al. [9] use histograms of oriented gradients for pedestrian detection based on infrared images. In this study, fixed histograms of gradients are mainly used.

In order to match the cloud vertical levels as determined by a commonly applied cloud remote sensing classification method (will be used to validate our method), the international satellite cloud climatology project (ISCCP), the cloud top pressure (CTP) data are associated with the CTT-base images. The CTP helps to divide clouds according to the atmospheric pressure level (i.e., the altitude at the top of the cloud) where they occur. Three cloud levels are distinguished here (high, middle, and low clouds). At each level, the edge detection technique is used on the CTT images to determine three cloud types (cirrus, cirrostratus, and deep convection for the high clouds; altocumulus, altostratus, and nimbostratus for the middle clouds; cumulus, stratocumulus, and stratus for the low clouds). Both, the CTT and CTP images, are extracted from thermal infrared observations of the national oceanic and atmospheric administrationadvanced very-high-resolution radiometer (NOAA-AVHRR) satellite afternoon ascending orbit (2 P.M.) by the pathfinder atmospheres extended (PATMOS-x) project. These images are daytime global data with a horizontal spatial resolution of $0.5 \times 0.5$ degree.

The choice of the CTT images to conduct this study is based on the capacity of the CTT to mimic the external shape of the cloud. A segmentation image technique expressed through edge detection analyses applied on CTT images (at each $3 \times 3$-pixel area) for cloud differentiation uses the frequency of occurrence of a local gradient histogram to finally distinguish cloud types. The histogram data size is expanded to $5 \times 5$-pixel gradient, in order to minimize noise contamination and increase higher separations between clouds.

To conduct the present cloud type classification study, the paper is organized as follows: subsequent to the introduction, the image segmentation concept for cloud types' differentiation will be presented. Then, the classification procedure will be described. In Section 4, the results and interpretation of the new cloud classification and the comparisons with a commonly used classification will be discussed. The study will end with a conclusion.

\section{Image Segmentation Concept for Cloud Types}

Image segmentation techniques permit grouping pixels into clusters representing prominent areas of the image and consequently different features. The cluster pixels correspond to separate individual and meaningful objects the human can visualize. Commonly used edge detectors are linear or nonlinear, first or second degree, or a combination of some of these. In image segmentation applications, various processing tools are used to detect edges or locate specific objects based on the radiance gradient of the image. Among these is the Canny edge detection [10]: it is one of the most commonly used tools; its implementation includes several steps among which is the integration of the Sobel edge gradient, used for the computation of the gradient magnitude and direction. The Sobel gradient, obtained from the Sobel [11] edge detection tool, uses the same number of pixels as the Prewitt gradient [12] but is more sensitive to diagonal edges compared to horizontal and vertical edges for the latter. The Roberts edge detector [13] uses fewer pixels than the previously cited tools but produces noisier features. All these edge detectors basically allow for the segmentation of the image in two major areas: edge and nonedge.

In this study nonlinear, first-degree, and second-degree detectors are tested and applied on CTT images for cloud type differentiation. The concept underlying the cloud types' differentiation proposed in this study is summarized in Table 1. In this table, clouds are separated into three pressure levels based on the CTP: high, middle, and low clouds. At each cloud level, the cloud external morphology will vary from structured to nonstructured clouds. The structured clouds are areas of high gradient while nonstructured clouds are areas 


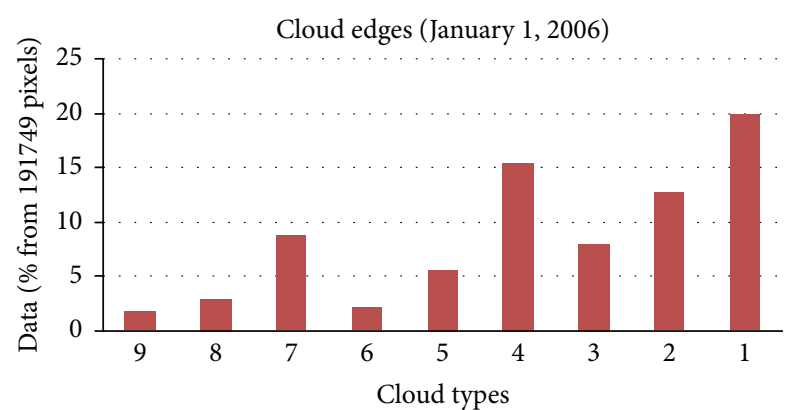

Figure 1: Edge distribution among the different cloud types. 1: cirrus, 2: cirrostratus, 3: deep convection, 4: altocumulus, 5: altostratus, 6: nimbostratus, 7: cumulus, 8: stratocumulus, and 9: stratus. The graph shows that the cirrus, altocumulus, and cumulus clouds are more likely to have edges than the other types of clouds. This histogram is based on NOAA-AVHRR CTT satellite images of the Globe at a spatial resolution of 0.5 degree. The gradients from which the cloud types are derived are associated with the corresponding areas of cloud top pressure images and matched with the cloud type classification map of a commonly used method in cloud remote sensing, the ISCCP method (based on the cloud optical depth and the cloud top pressure). The more edges exist in a specific area, the more the cloud encountered is structured.

of low gradient. High gradient areas are made of cumuluslike clouds (cirrus, altocumulus, and cumulus), low gradient areas are made of stratus-like clouds (deep convection, nimbostratus, and stratus), and intermediate gradient areas are made of intermediary clouds (cirrostratus, altostratus, and stratocumulus). Though, strictly speaking, cirrus clouds may be lacking structure, their limited spatial continuity (also CTT discontinuities) gives them the appearance of structured clouds on the CTT image. The more edges exist in a specific area, the more the cloud encountered is structured. Based on this concept, by using NOAA-AVHRR CTT derived satellite images of the Globe at a spatial resolution of $0.5 \times 0.5$ degree, the likelihood of edge occurrence for all cloud types based on an edge detection method (e.g., Sobel) for $3 \times 3$ array of pixels can be calculated as the gradient by

$$
\begin{gathered}
G_{x}=\frac{1}{8} \times\left|\begin{array}{lll}
-1 & 0 & +1 \\
-2 & 0 & +2 \\
-1 & 0 & +1
\end{array}\right| \quad G_{y}=\frac{1}{8} \times\left|\begin{array}{ccc}
+1 & +2 & +1 \\
0 & 0 & 0 \\
-1 & -2 & -1
\end{array}\right| \\
G=\sqrt{G_{x^{2}}+G_{y^{2}}},
\end{gathered}
$$

where $G_{y}$ and $G_{x}$ are the cloud top temperature gradients, respectively, in the $y$ and $x$ directions and $G$ is the final gradient.

This gradient is associated with the corresponding area of cloud top pressure and matched with the results of cloud type classification data commonly used in cloud remote sensing, the ISCCP method (based on the cloud optical depth and the cloud top pressure). As shown in Figure 1, the number of edges within each cloud level (low, middle, and high) drastically diminishes from the ISCCP identified, cumuluslike clouds (cirrus, altocumulus, and cumulus) to the stratuslike clouds (deep convection, nimbostratus, and stratus).
For example the most structured clouds in Figure 1 are 7: cumulus, 4: altocumulus and 1: cirrus. These clouds have the highest number of edges in their respective cloud levels. The groups are represented on the figure by numbers: 9,8 , and 7 for low clouds, 6, 5, and 4 for middle clouds, and 3, 2, and 1 for high clouds. Because of their altitude in the atmosphere, the total number of edges will decrease from high clouds to low clouds (more edges in the former than in the latter). Consequently, when using an edge method to distinguish clouds, we will need different edge threshold for each cloud level.

\section{Classification Procedure}

Morphologies of objects observed by remote sensing instruments can be extracted by using properties of the edges of the pixels of the imagery representing those objects. In this study, our focus is the shape of objects describing clouds, as obtained through the CTT images. The fundamental issue to deal with in this study is how to translate visual changes in CTT images to cloud features, significantly representative of different cloud types (see Introduction). The gradient concept presented in the previous section is implemented here. The initial step of this implementation is the calculation of the gradient magnitude representing the change in the CTT function of neighboring pixels of the image (e.g., the Sobel gradient calculation shown in the previous section). The calculated gradient map will undergo thresholding, in order to separate important features of the image at each cloud atmospheric pressure level (high, middle, and low). The thresholds applied are determined from the histogram distribution of CTT gradients of the image. Different thresholds will be applied to different cloud levels. Higher thresholds for the high clouds, medium for the middle clouds, and low for the low clouds.

A certain number of gradient operators using small arrays of pixels were tested in this study, in order to check their capacity to properly detect significant and meaningful boundaries between different CTT and cloud types eventually. This capacity is visually tested against direct CTT images. Among the detectors applied are the Canny, Roberts, Sobel, and Kayyali SENW (will be named from now on SENW) edge detectors, as well as the Harris corner and edge detector. The Roberts edge detector employs mainly 2 pixels in a $2 \times 2$ matrix for each computing direction (horizontal and vertical). The test conducted shows a lesser sensitivity to edges; that is, the variation range of gradients is limited compared to the other methods. The Canny method uses a $5 \times 5$-pixel matrix that undergoes preliminary filtering before the use of a smaller-array gradient detector, making the calculation procedure longer. The Sobel detector uses a $3 \times 3$ matrix where 6 pixels practically contribute to the gradient. The SENW edge detector is based on the Sobel approach for edge detection but employs mainly the 4 corner pixels (upper, lower, left, and right) in a $3 \times 3$-pixel matrix the remaining 5 pixels between the corners are set to zero. The Harris detector detects both edges and corners. It is a second-order derivative obtained from the calculation of 


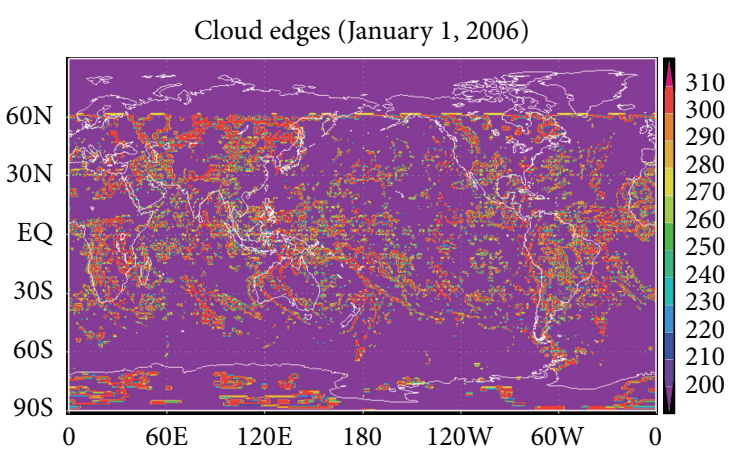

(a)

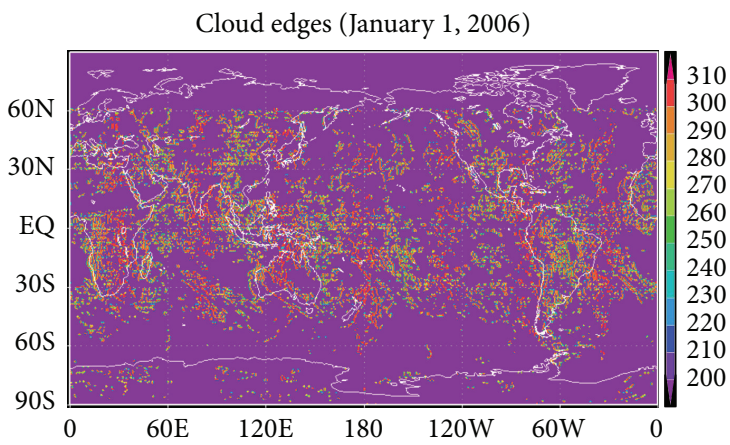

(c)

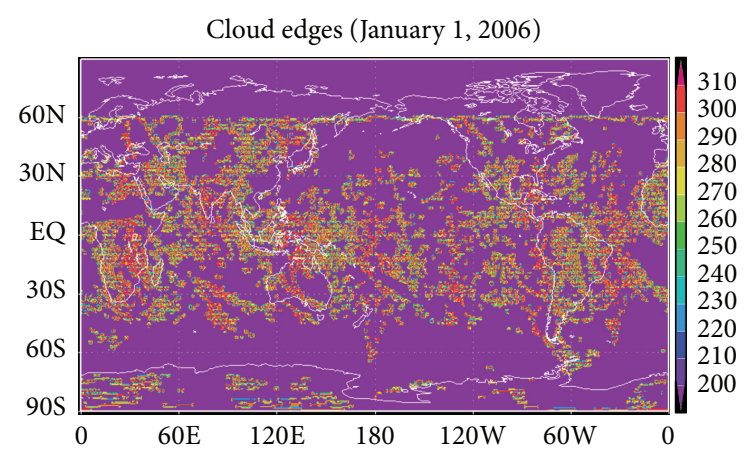

(b)

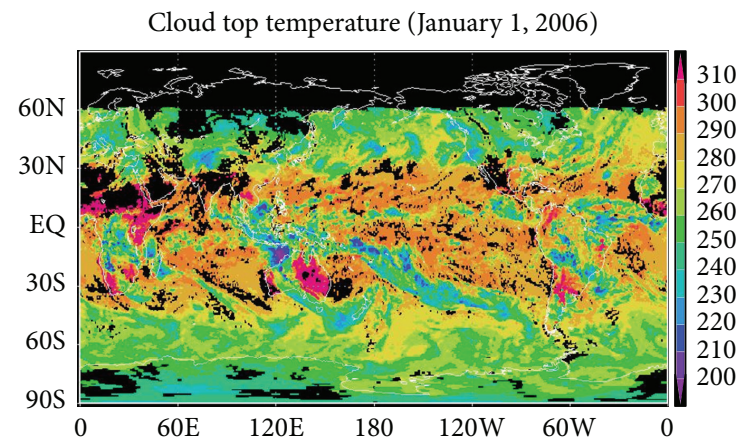

(d)

Figure 2: Detection of edges and corners by the Harris (a), SENW (b), and the Sobel (c) edge detectors; the colored CTT image (d) for visual comparison with the edge detectors. On the edge detectors images, the purple background represents nonmaxima or nonedges and the remaining colors represent maxima or edges and corners.

the Sobel gradients in the $x$ and $y$ directions then, the gradient covariance matrix $G$ [14]:

$$
G=\left|\begin{array}{cc}
I_{x}^{2} & I_{x} I_{y} \\
I_{x} I_{y} & I_{y}^{2}
\end{array}\right|
$$

where $I_{x}$ and $I_{y}$ denote the image gradients in the $x$ and $y$ directions and $I_{x} I_{y}$ denotes the sum of gradients in the $x$ and $y$ directions.

The corner response $R$ is expressed as [15] the ratio of the matrix determinant $\operatorname{det}(G)$ over the $\operatorname{trace} \operatorname{tr}(G)$ :

$$
R=\frac{\operatorname{det}(G)}{\operatorname{tr}(G)}
$$

Edges will have a negative $R$ value, while corners and interior points will have a positive $R$ value. In the latter case (positive $R$ ), interior points will have a very small $R$, while corner points will have higher $R$.

Due to their superior sensitivity to cloud detection, three methods (Sobel, Harris, and SENW detectors) among those cited above were selected, and their performance for cloud edges differentiation was tested on global images CTT maps obtained from NOAA-AVHRR satellite. Figure 2 presents the visual comparison between the results of these edge detectors using the CTT data and the latter colored image. This comparison shows that among the three detectors, the Harris detector seems to better represent corners and edges seen on the colored CTT cloud image than both the Sobel and the SENW detectors, but the density of these edges is lower than that of the latter detector. The Sobel misses some edges especially at the southern pole and at the boundary towards the northern pole (60N latitude). In the following step of our analysis, the results from these three detectors are compared to the results of the ISCCP cloud classification. Before this, let us first describe the detection steps for the cloud classification using these edge detectors. The flowchart in Figure 3 illustrates this description. It shows that the determination of the gradients is first made and then the histogram of gradients is calculated on each $5 \times 5$ pixel-gradient area. The midfrequency of the histogram will determine the type of cloud encountered. To determine this midfrequency, adaptive thresholding techniques are employed, that is, specific thresholds at each CTP $(<440 \mathrm{mb}$ for high clouds, $440-680$ for middle clouds, and $>680 \mathrm{mb}$ for low clouds) predetermined level. For each CTP level, there are 2 thresholds permitting the separation of high gradients from middle gradients (threshold 1) and then middle gradients from low gradient areas (threshold 2). Plane surfaces are likely to be stratus-like clouds while nonplane surfaces would mostly be cumulus-like clouds. The 50th percentile (midfrequency) of the histogram of gradients obtained at each pressure level will determine the cloud type at that level. If the 50th-percentile gradient of the $5 \times 5$-gradient matrix is 


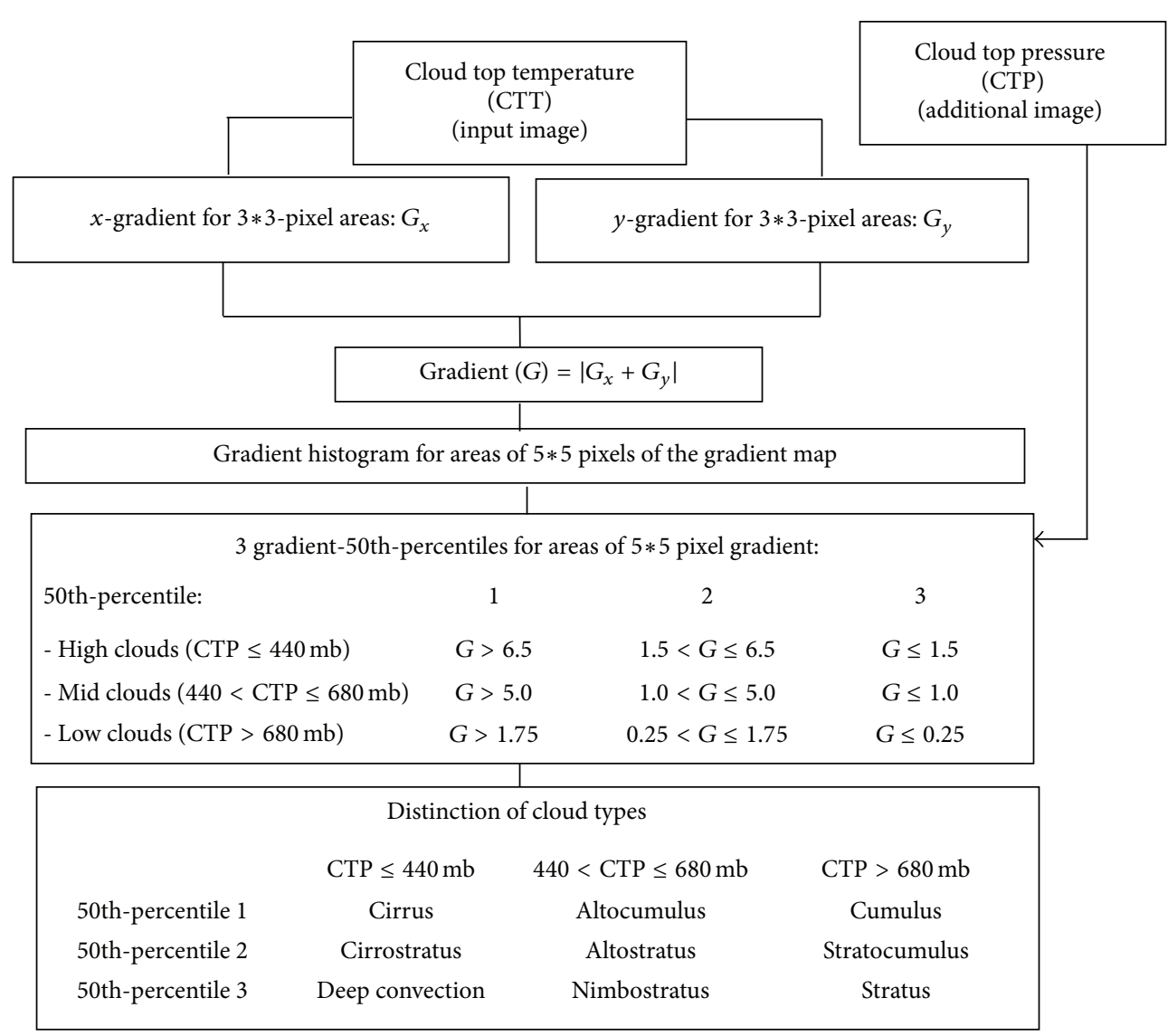

FIGURE 3: Flow chart of the thermal image segmentation for cloud type morphology differentiation based on the histogram CTT gradient method.

in the high gradient bracket, the central pixel is a cumuluslike cloud type (cumulus, altocumulus, and cirrus). If it is in the low gradient bracket, the central pixel is a stratus-like cloud type (stratus, nimbostratus, and deep convection). If it is in the intermediate gradient bracket, the central pixel is in the intermediary cloud type (stratocumulus, altostratus, and cirrostratus). We therefore distinguish three cloud types at each pressure level, as in the cloud optical properties based classification of the ISCCP $[2,16]$ : the high clouds are cirrus (Ci), cirrostratus (Cs), and deep convection (Dc); the middle clouds are altocumulus (Ac), altostratus (As), and nimbostratus $(\mathrm{Ns})$; the low clouds are cumulus $(\mathrm{Cu})$, stratocumulus (Sc), and stratus (St).

\section{Results and Interpretation}

The image segmentation technique applied on satellite CTT global images, described in this study, is used to differentiate cloud morphologies. The interpretation of these morphologies helps to obtain cloud types. As stated in the previous section, three edge detection methods were selected (Sobel, Harris, and SENW) and their capacity to detect important changes (edges) in CTT was assessed. Between the 3 methods used, the results of the cloud type spatial distribution are nearly similar: cirrus clouds dominate around the equator and most continental areas; cumulus and stratocumulus clouds are mainly visible in the ocean areas; stratus clouds mostly occur in the eastern part of the South Pole. All the 3 methods were tested in different weather conditions and seasons. The closest cloud classification to that of the ISCCP was obtained from the SENW based classification (2-3\% higher matching rate than the Harris, which is better by $1.5-$ $2 \%$ than the Sobel). Almost similar differences were obtained for these tests on images at all seasons. Figure 4 shows the comparison of the results obtained with the selected method against the CTT global image of January 1, 2006. The cloud type pattern exhibited by this method (named here CTT gradient method) appears quite close to that of the ISCCP. Many clouds in the southern hemisphere midlatitude tend to have an NW-SE orientation, contrary to the northern hemisphere midlatitude where the NE-SW orientation appears to dominate and the southern pole showing mainly E-W clouds. Cirrus/cirrostratus clouds are dominant near the equator, while nimbostratus and stratus are the main clouds near the South Pole, and cumulus/stratocumulus clouds are at midlatitude areas.

A detailed evaluation of the performance of this method (Figures 5 and 6) against the cloud optical depth based method from the ISCCP is discussed below. Figure 5 presents a qualitative evaluation of these results for a winter day (January 1), a spring day (April 2), and a summer day (September 3 ) of the year 2006. The figure shows that the 


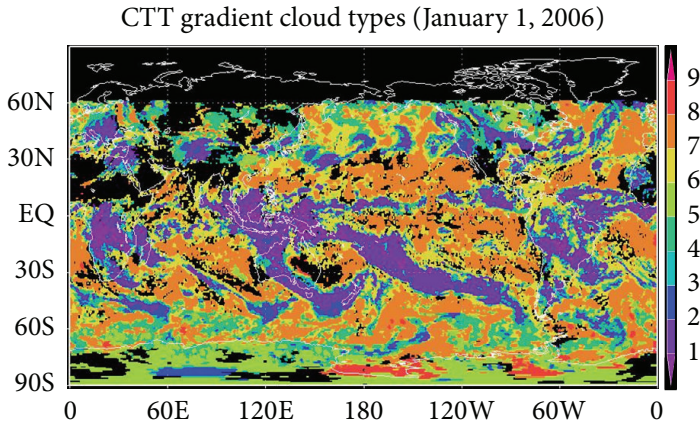

(a)

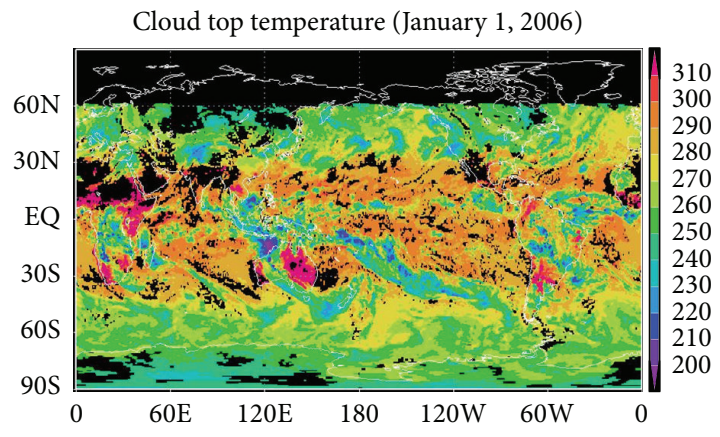

(b)

FIGURE 4: Cloud type classification using the cloud top temperature (CTT) gradient image segmentation algorithm (left) compared to the CTT colored image (in degree-K). 1: cirrus, 2: cirrostratus, 3: deep convection, 4: altocumulus, 5: altostratus, 6: nimbostratus, 7: cumulus, 8: stratocumulus, and 9: stratus.
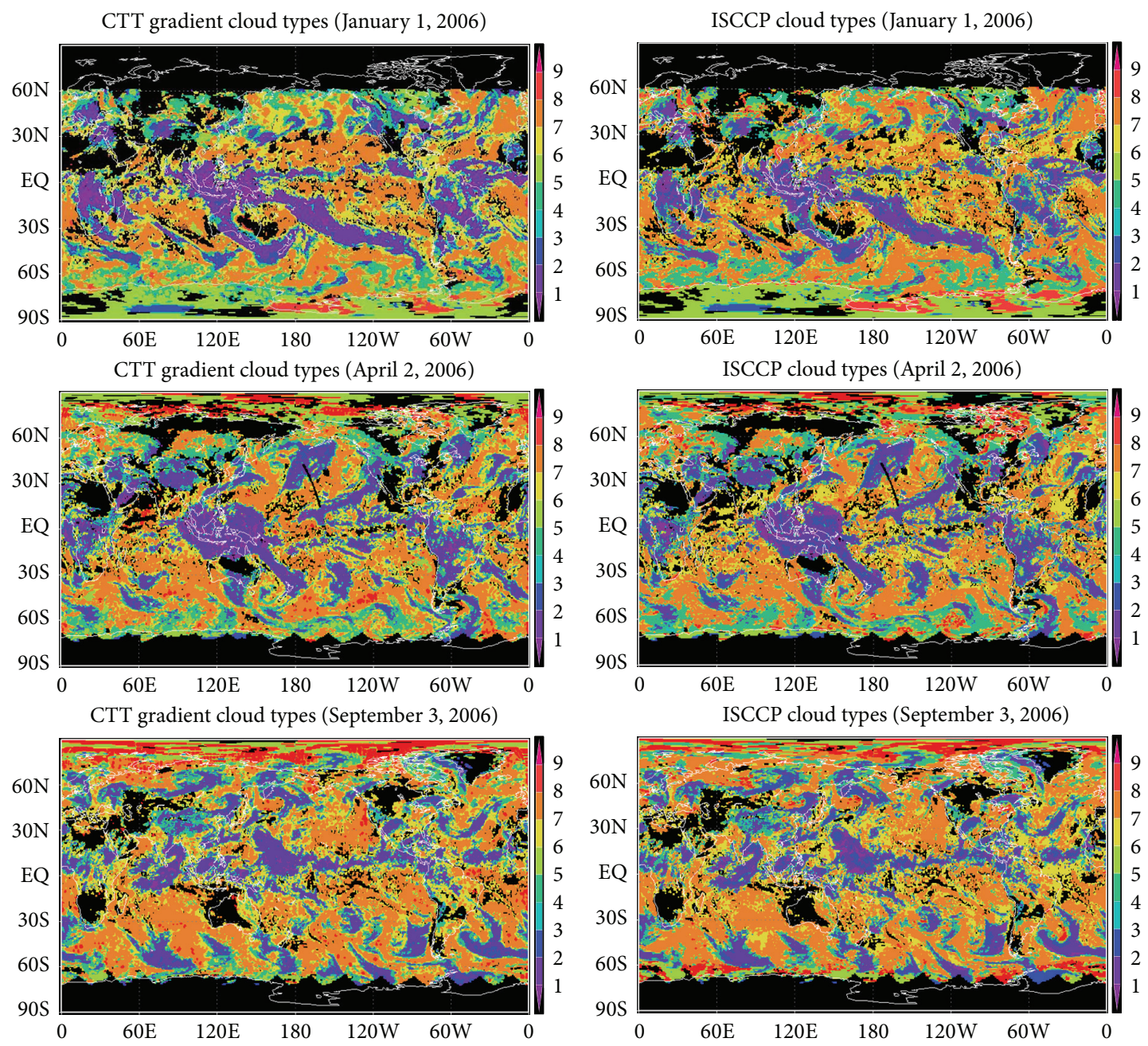

(a)

(b)

FIGURE 5: Cloud type classification using the thermal image segmentation method based on cloud top temperature (CTT) gradient classification (a) compared to the cloud type classification using the ISCCP classification method (b) for spring and end of summer (April 2 and September 3, 2006, resp.). 1: cirrus, 2: cirrostratus, 3: deep convection, 4: altocumulus, 5: altostratus, 6: nimbostratus, 7: cumulus, 8: strato-cumulus, and 9: stratus. 


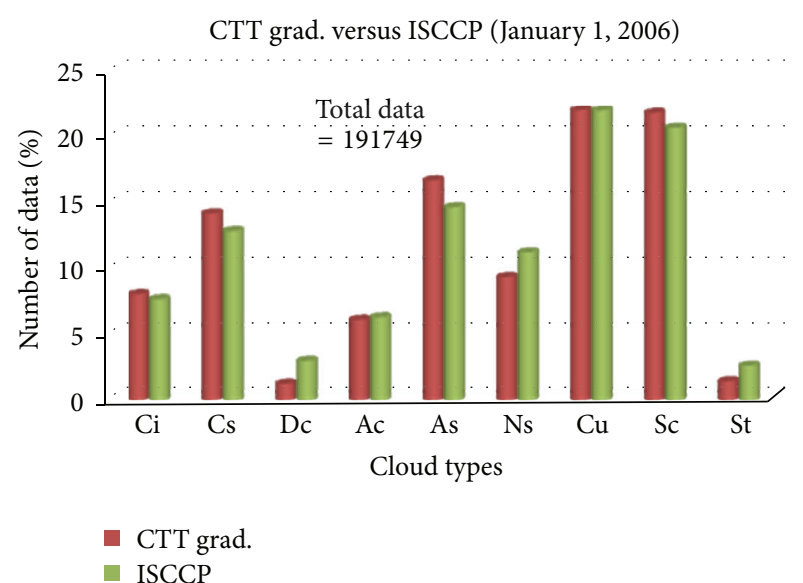

(a)

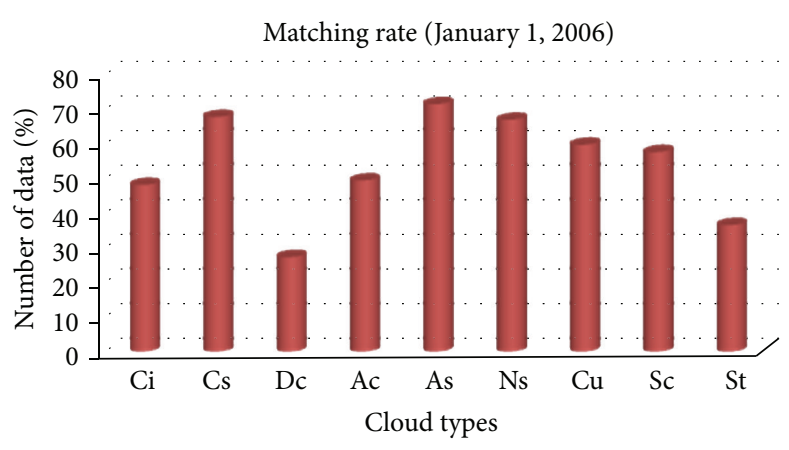

(b)

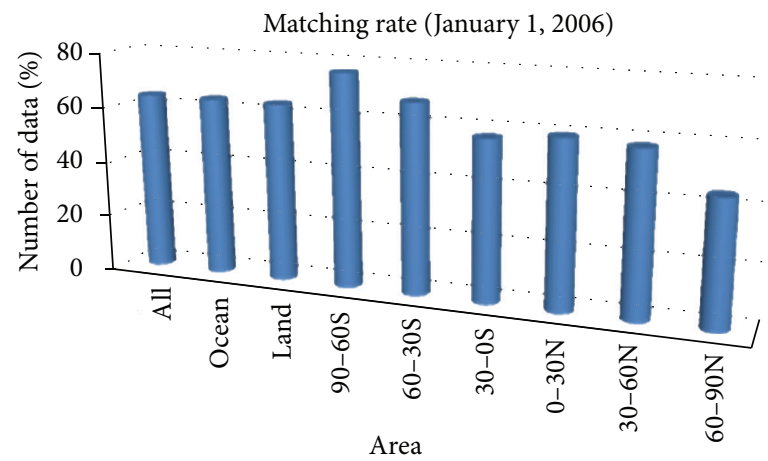

(c)

FIGURE 6: Comparative histograms for January 1, 2006, of the cloud type frequency obtained from the cloud top temperature (CTT) gradient classification a, b and the ISCCP classification method (a) the matching rate for each cloud type (b) and each region (c). 1-Ci: cirrus, 2-Cs: cirrostratus, 3-Dc: deep convection, 4-Ac: altocumulus, 5-As: altostratus, 6-Ns: nimbostratus, 7-Cu: cumulus, 8-Sc: stratocumulus, and 9-St: stratus. All: all areas.

cloud distribution pattern between the two methods is quite close with the best apparent matches at mid-structured clouds while more differences appear with the stratus-like clouds (blue, green, and red, respectively, corresponding to deep convection, nimbostratus, and stratus). At the spatial level, the southern pole shows apparent good matches. This is due to the strong occurrence of mid-structured clouds in this area. Land areas showed better matches than the ocean areas. Substantial differences mostly occur with deep convection, altocumulus and stratus clouds, where the edge detection method underestimates the frequency of these clouds. These clouds are mostly low gradient clouds and, because of the low resolution of the CTT images used, the spatial contrast is not good enough to efficiently separate these clouds from the rest of the clouds. Some other differences are visible in the South Pole with the detection of less stratus clouds (red on Figure 5) in the CTT gradient method. There are more cumulus (yellow) and fewer stratocumulus (orange) clouds between 30 and $60 \mathrm{~N}$ latitudes (January 1, 2006). The frequency and distribution of the cloud types obtained by the CTT gradient method, from one of the representative CTT images, Jan 1, 2006 (Figure 5) show the following: high clouds: cirrus (7\%), cirrostratus (15\%), and deep convection (1\%); middle clouds: altocumulus (3\%), altostratus (19\%), and nimbostratus (10\%); low clouds: cumulus (23\%), stratocumulus (20\%), and stratus (2\%). Figure 6 presents the comparative histogram of the cloud types' distribution between the CTT gradient method and the ISCCP method and their matching rate for each cloud type and for each major region. The CTT gradient method tends to underestimate the number of stratus-like clouds in favor of the other types of clouds. This is due (as stated before) to the relatively poor radiometric resolution of the CTT images and the presence of many gaps (black background) in the initial CTT image. The matching rate is the lowest among these clouds, mostly less than $50 \%$. The areal distribution shows that land areas produce better matches than the ocean areas. The regional distribution shows that except for the 60 $90 \mathrm{~N}$ latitude area, where there are very few data, the lowest matching rate is in the $0-30 \mathrm{~S}$ latitude region. This is due to the relatively large presence of deep convection clouds (dark blue in Figure 5) in that area. The CTT gradient shows considerable difficulties in the detection of these types of clouds. Overall, monthly average global cloud distributions gave relatively good agreements between the two methods ( 60 to $70 \%$ ) for the year 2006 . 


\section{Conclusion}

An alternative cloud type classification method to commonly used cloud remote sensing methods is proposed in this study. This classification is based on the application of edge detection techniques on CTT global images. Areas of high gradient correspond to cumulus-like clouds, while low gradient areas are mostly associated with stratus-like clouds. Various gradient operators using small arrays of pixels are tested, in order to check the detection capacity of important boundaries between different CTT and cloud types eventually. This capacity is visually tested against direct CTT images. The detectors applied include the Canny, Roberts, Sobel, and SENW edge detectors, as well as the Harris corner and edge detector. This detector list is narrowed to three after preliminary tests. The method that detected the most edges gave the best results in the final cloud classification. The validation of these methods is made through a comparison with a commonly used cloud remote sensing method, the ISCCP method (based on the cloud optical properties). The closest cloud classification to that of the ISCCP is obtained from the SENW based classification with around 3\% and $5 \%$ higher matching rate than the Harris and the Sobel methods, respectively. The success rate of the best method is not seasonally dependent as the differences with the ISCCP method are almost similar at any time of the year. And, in general, there are relatively good matching rates of about 60 to $70 \%$. Among the cloud types, the best matches were obtained with the mid-structured clouds, while the lowest were with the stratus-like clouds. At the spatial level, the southern pole showed the best matches as in this area there is a strong occurrence of mid-structured clouds (more easily detectable by the edge gradient method). Land areas showed better matches than the ocean areas. The CTT gradient method used may be refined and additional edge detectors tests could lead to the improvement of the results obtained.

\section{Acknowledgment}

The authors are very grateful to the PATMOS-x project for providing the global NOAA-AVHRR images used in this study.

\section{References}

[1] J. R. Dim, H. Murakami, T. Y. Nakajima, B. Nordell, A. K. Heidinger, and T. Takamura, "The recent state of the climate: driving components of cloud-type variability," Journal of Geophysical Research D, vol. 116, no. 11, Article ID D11117, 2011.

[2] W. B. Rossow and R. A. Schiffer, "Advances in understanding clouds from ISCCP," Bulletin of the American Meteorological Society, vol. 80, no. 11, pp. 2261-2287, 1999.

[3] T. Inoue, "A cloud type classification with NOAA 7 split-window measurements," Journal of Geophysical Research, vol. 92, no. 4, pp. 3991-4000, 1987.

[4] N. Dalal and B. Triggs, "Histograms of oriented gradients for human detection," in Proceedings of the IEEE Computer Society Conference on Computer Vision and Pattern Recognition (CVPR '05), vol. 1, pp. 886-893, June 2005.
[5] N. Dalal and B. Triggs, "Histograms of oriented gradients for human detection," in Proceedings of the IEEE Computer Society Conference on Computer Vision and Pattern Recognition (CVPR '05), vol. 1, pp. 886-893, June 2005.

[6] D. Sen and S. K. Pal, "Histogram thresholding using fuzzy and rough measures of association error," IEEE Transactions on Image Processing, vol. 18, no. 4, pp. 879-888, 2009.

[7] S. M. Smith and J. M. Brady, "SUSAN-a new approach to low level image processing," International Journal of Computer Vision, vol. 23, no. 1, pp. 45-78, 1997.

[8] A. Shashua, Y. Gdalyahu, and G. Hayun, "Pedestrian detection for driving assistance systems: single-frame classification and system level performance," in Proceedings of the IEEE Intelligent Vehicles Symposium, pp. 1-6, June 2004.

[9] F. Suard, A. Rakotomamonjy, A. Bensrhair, and A. Broggi, "Pedestrian detection using infrared images and histograms of oriented gradients," in Proceedings of the IEEE Intelligent Vehicles Symposium (IV '06), pp. 206-212, June 2006.

[10] J. Canny, "A computational approach to edge detection," IEEE Transactions on Pattern Analysis and Machine Intelligence, vol. 8, no. 6, pp. 679-698, 1986.

[11] I. Sobel, "An isotropic $3 \times 3$ gradient operator," in Machine Vision for Three-Dimensional Scenes, H. Freeman, Ed., pp. 376-379, Academic Press, New York, NY, USA, 1990.

[12] J. M. S. Prewitt, “Object enhancement and extraction," in Picture Processing and Psychopictorics, Academic Press, 1970.

[13] L. Roberts, Machine Perception of 3-D Solids, Optical and Electro-Optical Information Processing, MIT Press, 1965.

[14] C. Harris and M. Stephens, "A combined corner and edge detector," in Proceedings of the 4th Alvey Vision Conference, pp. 147-151, Manchester, UK, 1988.

[15] A. Noble, Descriptions of image surfaces [Ph.D. thesis], Department of Engineering Science, Oxford University, 1989.

[16] W. B. Rossow, A. W. Walker, and L. C. Garder, "Comparison of ISCCP and other cloud amounts," Journal of Climate, vol. 6, no. 12, pp. 2394-2418, 1993. 

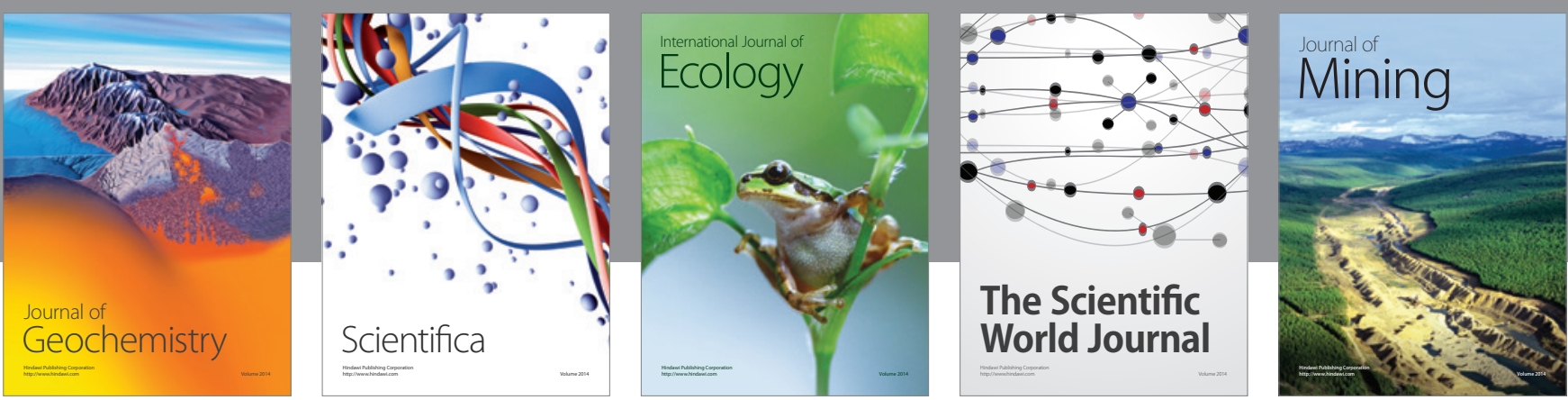

The Scientific World Journal
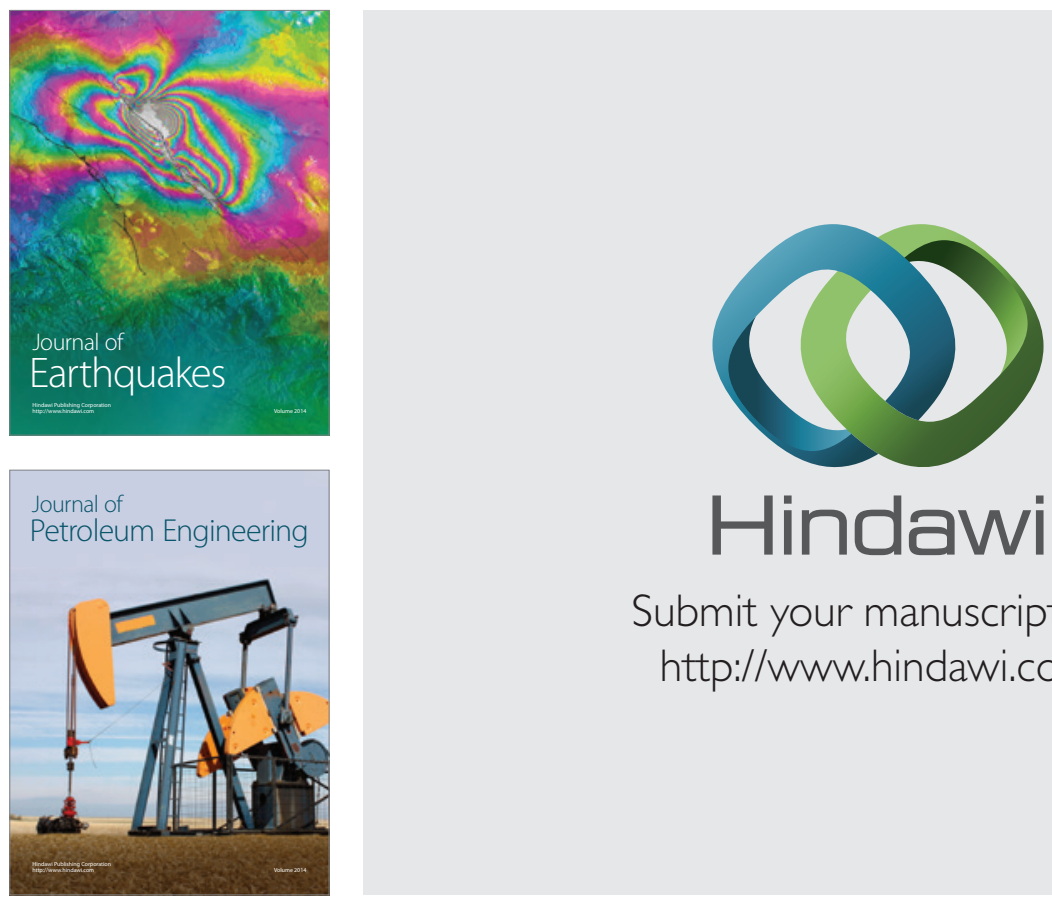

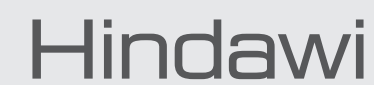

Submit your manuscripts at

http://www.hindawi.com
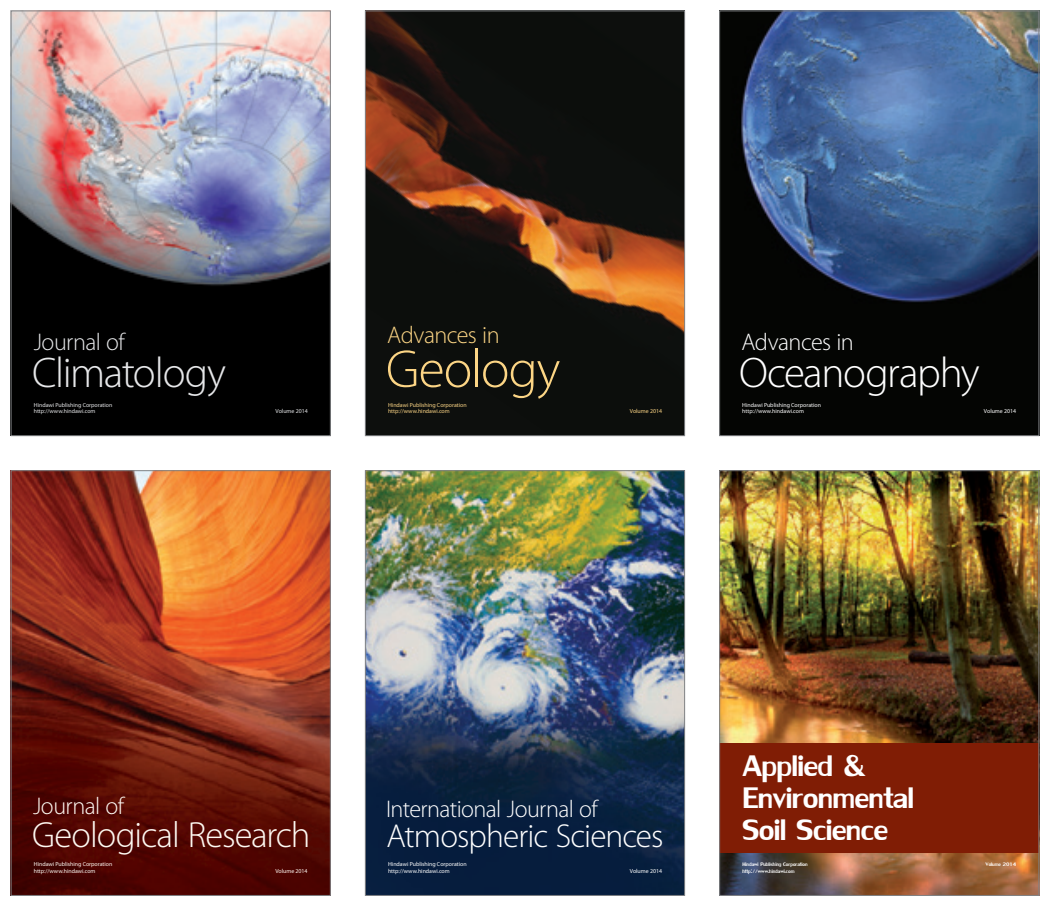
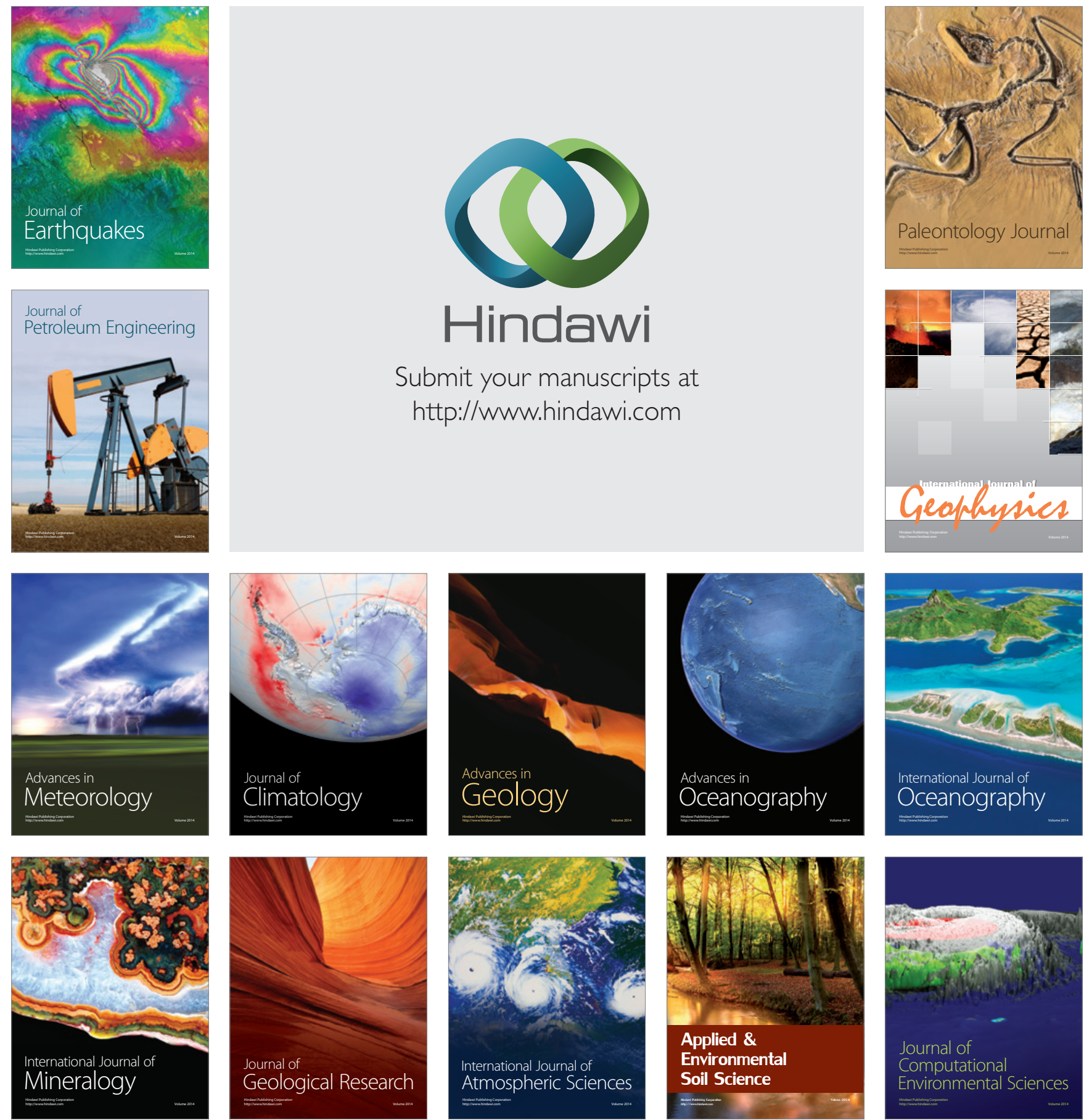